\title{
Matching of facial features: Continuous processing, improper filtering, and holistic comparison
}

\author{
ISTVÁN CZIGLER \\ Institute for Psychology, Budapest, Hungary
}

\begin{abstract}
The aim of the present study was to investigate the effect of irrelevant features on reaction time (RT) in a matching task, where the relevant and irrelevant features are parts of a well established unit, that is, the human face. In Experiment 1, subject had to decide whether successively presented pairs of photographed faces were the same or different with respect to relevant features (eyes or hair). As a function of the number of irrelevant different features (from zero to three) the "same" RT increased. The rate of increase was larger when the relevant feature was eyes rather than hair. RT was shorter in the hair stimulus condition than in the eye stimulus condition. "Same" RT was longer than "different" RT. In Experiment 2, the facial features were presented alone. RT for the hair was shorter than for eyes. In the hair stimulus condition, the "same" RT appeared to be shorter than the "different" RT, whereas the opposite result was obtained for the eyes. In Experiment 3, the main results of Experiment 1 were replicated in a procedure in which a "standard" face was continuously presented during an experimental run. Although no single model constructed for the matching tasks can explain all the data, the response competition approaches, supplemented by the possibility of stimulus set selection, and the contribution of holistic facial representation might be interpreted as accounting for the results.
\end{abstract}

When subjects judge stimulus pairs to be the same or different, "same" responses are typically made faster than "different" responses. Theoretical accounts of this phenomenon have made an important contribution to our knowledge about human information processing. The majority of the matching phenomena were obtained in experiments in which the stimuli were letters from the English alphabet, digits, and geometrical forms. In the present study, our aim was to investigate the explanatory power of the different models of the matching task using a kind of stimulus material that was supposed to be familiar to the subjects. Photographs of the human face were used. In the case of such stimuli, hemispheric studies (see Sergent \& Bindra, 1981, for a review) have emphasized the role of both holistic and analytic processing strategies.

In one set of explanations of the matching phenomena, the "fast-same" effect is attributed to qualitatively different processes underlying the two types of responses. Usually, a fast, "holistic" process is hypothesized in the case of "same" responses, whereas a slower, "analytic" process is hypothesized as mediating the "different" ones (e.g., Bamber, 1969). In one version of this approach, the processing consists of two stages, that is, there is a fast, but less accurate, stage and a slow, more careful,

I am indebted to Gábor Szily for the preparation of the stimulus material. I would like to thank Robert Proctor for his helpful comments on a previous version of this manuscript. Requests for reprints should be sent to I. Czigler, Institute for Psychology, Hungarian Academy of Sciences, Budapest, Pf. 398, H-1394, Hungary. one (Kroll \& Hershenson, 1980). The dichotomy of holistic and analytic processes is frequently connected with the different processing strategies presumed for the two hemispheres (e.g., Bagnara, Boles, Simion, \& Umiltà, 1983; Cohen, 1972; Patterson \& Bradshaw, 1975; Sergent, 1982).

The "two-process" framework has generally been accepted in matching studies with facial stimuli. Using facial stimuli in "same"'."different" reaction time (RT) tasks, there were no RT differences observed in the case of simultaneous (Moscovitch, Scullion, \& Christie, 1976) or successive stimulus delivery with zero interstimulus interval (Sergent, 1982). Using a longer interstimulus interval, however, the "same" RT appeared to be shorter than the "different" RT for schematic faces (Patterson \& Bradshaw, 1975), line drawings of faces (Moscovitch et al., 1976), and photographs (Suberi \& McKeever, 1977) as well.

In another set of explanations, the "fast-same" phenomenon has been attributed to the different treatment of the same and different pairs by the processing mechanism. In the "noisy operator"' theory, Krueger (1978) considered the internal noise of the encoding processes as the cause of the fast "same" response. According to this model, in some "same" trials, a high difference count appears to be an effect of the random noise. Thus, a fast "same" response can be initiated whenever the result of the difference counting is very low. In order to avoid erroneous "different" responses, then, it is necessary to recheck whenever the different count exceeds the low criterion. 
In a modified version of this theory, Miller and Bauer (1981) suggested that instead of the hypothesized noisy representation of the stimuli, attributable to improper filtering, the locus of the noise effect is the comparison mechanism. On certain "same" trials, the irrelevant differences are successfully filtered out and, consequently, a new pass can be avoided. The "fast-same" phenomenon is the result of these cases, because the "different" responses are always preceded by the rechecking process. Miller and Bauer argued that external noise (i.e., irrelevant features of the stimuli) might eliminate the advantage of the "same" RT if a low level of representation was checked and the advantage of the "same" response was due to the low difference count. However, according to the data reported by Miller and Bauer, in the case of geometric stimuli, the fast "same" RT was preserved even in the presence of irrelevant differences between the members of stimulus pairs. On the other hand, Dixon and Just (1978) reported faster "different" RT when geometric stimuli had both task-relevant and irrelevant dimensions.

In another set of explanations, inhibitory processes, caused by the competition between the "same" and "different" responses, are considered as a source of the RT differences. According to Proctor (1981), there are two recognition responses in the "different" trials, while there is only one in the "same" trials. The relative slowness of the "different" responses is caused by mutual inhibition between the recognition responses.

The inhibition account of the matching phenomenon is also a basic element of the continuous flow model suggested by C. W. Eriksen, O'Hara and B. Eriksen (1982). According to this account, the usual "fast-same" effect is due to the priming of the "same" response in the "different" trials. In this model, the results of the encoding processes are continuously transferred toward the output organization. Early in the growth of the percept there is no difference discovered between the stimulus alternatives. Consequently, in this period of time, even in the "different" trials it is the "same" response that gets priming. The effect of this priming is the inhibition of the alternate ("different") response.

The continuous flow model is supported by results obtained in experiments in which irrelevant elements of visual stimuli were manipulated in "same"'-"different" RT tasks (Eriksen et al., 1982). The main variable in this study was the similarity between the irrelevant and target elements. In this situation, the irrelevant elements might actually modify the relationship between the "same"' and "different"' RT. Similar target and irrelevant letters had facilitating effects, whereas dissimilar irrelevant letters had inhibitory effects on the "same" RT. The opposite effects appeared on the "different" RT. An important feature of the continuous flow model is that it accounts for the instances in which the "different"' RT is shorter than the "same," provided that, in the particular experiment, there is the possibility of priming two "different" responses. In fact, in the presence of irrelevant disturbing elements, the "same"' RT was longer than the "different"' RT without the presence of irrelevant letters (Eriksen et al., 1982).

In various accounts of the matching phenomenon, some common elements are noticeable. According to the noisy operator model (Krueger, 1978) and the continuous flow theory (Eriksen et al., 1982), the locus of the "same"." "different"' RT deviance is an early level of the processing. In contrast, according to the improper filter approach (Miller \& Bauer, 1981) and the implicit naming interference theory (Proctor, 1981), the source of the RT differences is supposed to be the higher levels of encoding processes. According to the noisy operator theory (Krueger, 1978) and the two-stages theory (Kroll \& Hershenson, 1980), in the case of certain stimuli more than one pass of evaluation is needed. However, according to the former approach, these processes are similar, whereas in the latter model qualitatively different processes are hypothesized.

Complex and familiar stimuli such as the human face may provide an insight into the effects of different variables involved in the matching task. The "Gestalt-like" character of the human face makes this kind of stimulus suitable for the investigation of the validity of the models based on data obtained in experiments with irrelevant stimulus features. The main variable in the present study is the similarity between the irrelevant parts of the faces to be matched. As a function of the number of identical features, a decrease of "same" and an increase of "different" RT is expected on the basis of the response inhibition models (Eriksen et al., 1981; Proctor, 1981). In order to manipulate the relevant and irrelevant features, either the eyes or the hair was chosen as the task-relevant elements; the other parts of the face were regarded as potentially distracting features. As indicated by the low performance of facial reconstruction studies (Christie \& Ellis, 1981; Ellis, Shepherd, \& Davies, 1975), it is difficult to attend to a particular facial feature in the presence of the whole face.

Concerning the two task-related features, it turns out that eyes are one of the most salient parts of the face. In various studies, the eyes were judged as the most important part of the face (Laughery, Alexander, \& Lane, 1971), changes in the eyes were more easily detected than changes in the nose (Davies, Ellis, \& Shepherd, 1977), and the frequency of fixations concentrated on the eyes was greater than that on any other part of the face (Yarbus, 1967). Since the eyes are located within the face, it is difficult to "filter out" the other parts of the face by simple peripheral methods, such as slight changes of fixation. In this respect, selective processing of the hair is simpler. Furthermore, the hair has a definite and closer contour. As a consequence, hair may be processed more easily as an independent whole.

\section{EXPERIMENT 1}

\section{Method}

Subjects. The subjects were 20 undergraduate students of Eötvös Loránd University, Budapest. Participation in the experiment par- 
tially fulfilled a course requirement in experimental psychology.

Stimuli. Stimuli were selected from a large collection of photographs of hair, eyes, noses, and mouths. Four pairs of these features were selected. The features within a pair were easily discriminable, as evaluated by two raters. No formal procedure was used to assess discriminability quantitatively. The feature pairs were used in two conditions. In the first, the target feature was the eye; in the second the target feature was hair. One member of each pair was used to construct a standard face, while other members of the feature pairs were distractors. For the "same" trials of the experiment, faces were constructed from the standard member of the target feature pair, plus one, two, or three distractor features (the other features of the photographs were standard features). For the "different" trials, faces were constructed from the nonstandard members of the target features plus one, two, or three distractor features (the other features of the photographs were standard features). In this fashion eight sets of slides were constructed-four for the eye stimulus condition and four for the hair stimulus condition. In each set there were 24 pairs of slides. The first member of a pair was a standard face. There were 12 "same" and 12 "different" pairs in each set. As for the second member of the "same" pairs, there were three slides with either four, three, two, or one (the target) standard features, while among the second member of the "different" pairs, there were three slides with three, two, one, or zero standard features. In Figure 1, specimens of a set from the eye stimulus condition are shown.

Apparatus and Procedure. From the four sets of a given (eye or hair) stimulus condition, 96 stimulus pairs were constructed. The order of the pairs was randomized for each subject. These stimulus pairs were divided into three groups of 28,34 , and 34 pairs. The first group of stimulus pairs was supplemented by eight practice pairs (four "same" and four "different," randomly chosen from the 96 pairs), while the second and third groups were supplemented by two practice pairs (one "same" and one "different," randomly chosen from the 96 pairs). In this way, three runs of 36 trials were constructed.
The subjects were seated in a dimly illuminated room. The stimuli were presented using a Kodak Carousal slide projector equipped with a tachistoscopic shutter (Getra). Stimuli were rear-projected onto a translucent screen. From the $250-\mathrm{cm}$ viewing distance, the faces subtended $3^{\circ}$ horizontally and $4^{\circ}$ vertically. The luminance of the stimuli was $30 \mathrm{~cd} / \mathrm{m}^{2}$.

At the start of the trials, a warning click was presented. After $1,000 \mathrm{msec}$, the first member of the pair was presented for 3,000 msec, followed by the 3,000-msec presentation of the second member of the pair. The interstimulus interval was $3,000 \mathrm{msec}$.

Responses were made by the left and right index fingers' pressing one of two microswitches. Half of the subjects responded with the right hand when they decided the target feature of the pair was "same," and the other half of the subjects used the right hand to make "different" responses. RT was measured from the onset of the second member of the pairs, using a millisecond timer.

Each subject served in two identical sessions that took about $40 \mathrm{~min}$. Since the first session was considered as a practice session, only the results of the second session are reported here. For onehalf of the subjects, the target feature was the eye; for the other half, the target feature was the hair.

\section{Results}

Since the responding hand exhibited no effects on the RT and errors, in respect to this factor, the data were collapsed. Separate two-way ANOVAs were calculated for the "same" and "different" responses. Stimulus conditions (hair or eye) was a between factor, while the number of different features (four levels) was a within-subject factor. As for the "same" responses, both main effects were significant $[F(1,18)=6.11, p<.05$, for the stimulus conditions; $[\mathrm{F}(3,54)=67.63, \mathrm{p}<.01$, for the number of different features]. There was a significant interaction as well $[\mathrm{F}(3,54)=10.56, \mathrm{p}<.01]$. According
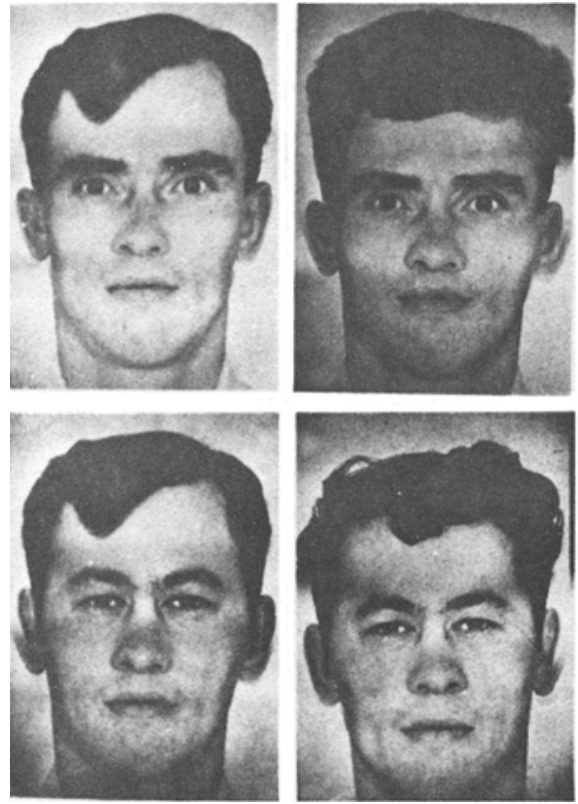
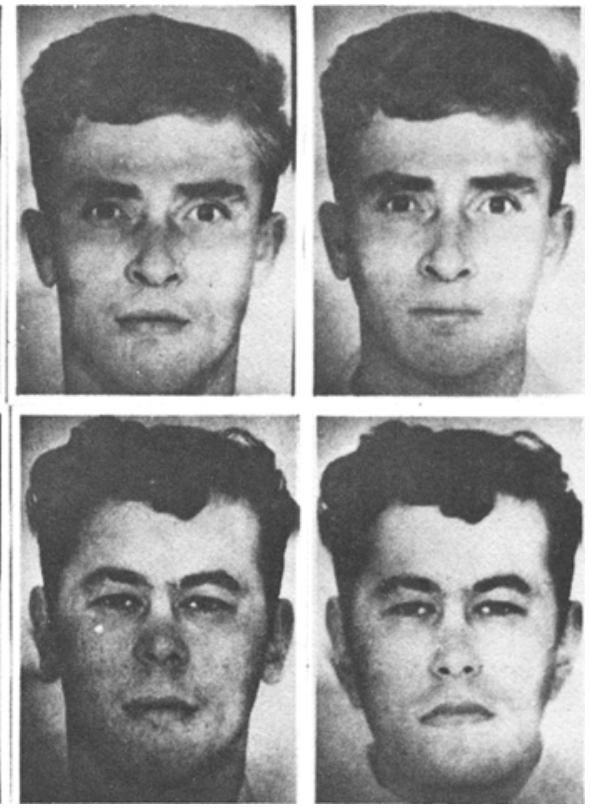

Figure 1. A sample of the stimulus faces from the eye stimulus condition of Experiments 1 and 3. The "standard" stimulus is shown on the left side of the upper row. The other three faces in the upper row are "same" faces with 1, 2, and 3 irrelevant different features (from left to right, respectively). In the lower row, "different" stimuli are shown with $0,1,2$, and 3 irrelevant different features (from left to right, respectively). 
to a similar two-way ANOVA of the "different" RT, no significant effects were found. On Figure 2, the average "same" and "different" RT data are shown from the two stimulus conditions, as a function of the number of different features. As shown in Figure 1, "same" RT is generally longer in the eye stimulus condition. As function the number of different features, the RT increases, and the rate of increase is larger in the eye stimulus condition.

In order to assess the effect of the different features, separate one-way ANOVAs were calculated for the "same" responses of the two stimulus conditions [for the eye stimulus condition, $F(3,27)=58.38, p<.01$; for the hair stimulus condition, $F(3,27)=14.92$, $p<.01]$. In both cases, only the linear trend appeared to be significant [for the eye stimulus condition, $F_{\text {lin }}(1,27)=$ $171.47, \mathrm{p}<.01$; for the hair stimulus condition $\mathrm{F}_{\mathrm{lin}}(1,27)$ $=44.37, \mathrm{p}<.01]$.

"Same" and "different" RT data were compared using a two-way ANOVA, with stimulus condition (between group) and response type (within subject) as factors. The main effect of stimulus condition was marginally significant $[F(1,18)=3.90, p<.07]$. The main effect of response type and the interaction were significant $[F(1,18)$ $=27.95, \mathrm{p}<.01 ; \mathrm{F}(1,18)=9.65, \mathrm{p}<.01$, respectively]. Thus, the "different" responses were faster and, in the eye stimulus condition, the RT was slower than in the hair stimulus condition. The "same"-"different" RT difference was larger in the eye stimulus condition.

In Table 1, the mean error percentages are shown. Because of the large number of zero values, the data were

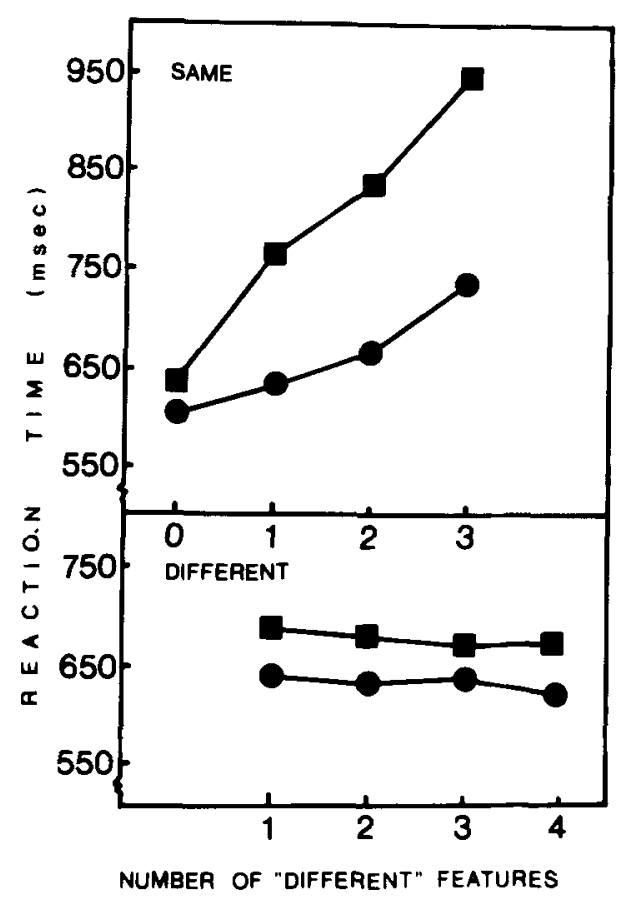

Figure 2. Mean "same" and "different" RT as a function of the number of different features in Experiment 1. $\square-0$, eye target; $\bullet-\bullet$, hair target.
Table 1

Percentage Error in Experiment 1

\begin{tabular}{|c|c|c|c|c|c|c|c|c|}
\hline \multirow[b]{3}{*}{ Stimulus Condition } & \multicolumn{4}{|c|}{ Same } & \multicolumn{4}{|c|}{ Different } \\
\hline & \multicolumn{4}{|c|}{ Different Features } & \multicolumn{4}{|c|}{ Different Features } \\
\hline & $\overline{0}$ & 1 & 2 & 3 & 1 & 2 & 3 & $\overline{4}$ \\
\hline Eye & 4.9 & 8.6 & 7.6 & 9.5 & 10.8 & 10.5 & 11.2 & 9.7 \\
\hline Hair & 3.5 & 7.8 & 5.1 & 5.8 & 5.0 & 7.4 & 3.3 & 3.3 \\
\hline
\end{tabular}

collapsed across the number of different features. Thus, a two-way ANOVA was calculated from the number of errors with stimulus conditions (between group) and response type (within subject) as factors. Only the main effect of stimulus type was significant $[\mathrm{F}(1,18)=35.61$, $\mathrm{p}<.01]$, indicating a smaller error rate for the hair stimulus condition.

\section{Discussion}

Irrelevant features have marked effects on the "same" $\mathrm{RT}$, that is, as the number of different irrelevant features increased, the "same"' RT increased. In this respect, the results are in accord with the response inhibition theory (Proctor, 1981) and the continuous flow model (Eriksen et al., 1982). According to the inhibition principle, the role of the similar elements would be to prime the "same" responses and the different irrelevant element would prime the "different" responses. However, this theory predicts the change of the "different" responses as well. In order to maintain the explanations in the framework of the inhibition models, the possibility of a "floor effect" must be considered with respect to "different" responses. In fact, the "different" RT was shorter than the "same" RT, and even the shortest "same" RT (identical members of the pair) was no shorter than the average "different" RT. ${ }^{1}$

Although in certain "same"-" different" RT tasks in the presence of irrelevant features, the "different" RTs become shorter than the "same" RTs (Dixon \& Just, 1978), in other studies, a maintained "same" RT advantage was found (Miller \& Bauer, 1981). It should be emphasized, that the response competition accounts (Eriksen et al., 1982; Proctor, 1981) do not predict the necessary reversal of the "same"'-"different" relationship in the presence of irrelevant features. The reduction of the "fast-same" effect is in accord with this model, and it was the result of the experiments reported by Miller and Bauer (1981).

Concerning the lack of the effect of the number of different irrelevant features on the "different"' RT, one might argue that, in spite of the manipulation of the magnitude of difference, these stimuli were equally different from the standard. The results of a similarity scaling experiment disproved this assumption. As a function of the number of different features, the "same" and the "different" pictures were rated as more and more different from the standard. ${ }^{2}$

As function of the number of different irrelevant features, a linear "same" RT increase was obtained. This finding rules out the two-process accounts (Bamber, 
1969). According to this model, because of the possibility of the fast "holistic" comparisons, the "same" RT would be disproportionately shorter in case of zero irrelevant difference. In addition, this RT would be shorter than the average "different" RT. These expectations receive no support from the present data.

Concerning the two-stage model (Kroll \& Hershenson, 1980), it is reasonable to suppose that, in the present experiment, the responses were always preceded by the working of the second stage, that is, by the analytic processes. The obtained relationship between the "same" and "different" RT is in conformity with assumptions concerning this kind of processing. However, on the basis of a strict feature-by-feature comparison process, restricted to the relevant stimulus dimension, no effect of the irrelevant different features is expected.

As Miller and Bauer (1981) noted, it is difficult to filter out irrelevant features in the case of multidimensional stimuli. An effect of improper filtering is the overloaded (noisy) decision mechanism, and consequently a longer RT. Although it is easier to filter out the irrelevant features in the hair stimulus condition, the preceding argument can be used to explain the different magnitude of the irrelevant feature effect on both stimulus conditions. On the other hand, the "noisy detection" model (Miller \& Bauer, 1981) does not predict gradual RT increase as function of the number of different irrelevant elements. Because of the possibility of the low difference count, this model would predict disproportionately faster "same" RT in cases of identical faces, much like the two-process account (Bamber, 1969).

On the basis of the present data, it is impossible to determine whether the interaction between the stimulus conditions and response types was due to the easier discrimination in the hair stimulus condition than in the eye stimulation condition, or the face as a whole, may have a more pervasive effect on the discrimination of the eyes.

\section{EXPERIMENT 2}

The aim of this experiment was to investigate whether, in the case of the presentation of isolated facial features, the "different" RT was shorter than the "same," as found to be the case in the presence of other parts of the face. Using line drawings of isolated facial features, Sergent (1982) reported no differences in "same" and "different" RT. Furthermore, the face as a whole may have larger effects on the eyes than on the hair. The source of the RT advantage of the hair-stimulus condition of Ex- periment 1 might, then, be interpretable as more than the mere discriminability of the two features.

\section{Method}

Subjects. The subjects were 16 undergraduate students of Eötvös Loránd University, Budapest. Participation in the experiment partially fulfilled a course requirement in experimental psychology.

Apparatus and Procedure. The stimulus material consisted of the eyes and hair of the faces used in Experiment 1. They were presented as isolated features in the center of a screen. Stimuli subtended $3^{\circ}$ horizontally and $2^{\circ}$ vertically from the $250-\mathrm{cm}$ viewing distance. In all other respects (i.e., the timing of the stimulation, the construction of the experimental runs, and the measurement of the responses), the procedure was the same as that used in Experiment 1.

Half of the subjects responded with the right hand when they decided the stimuli were "same," and the other half responded with the right hand for "different" 'responses. For half of the subjects, the stimuli were eyes, and for the other half, they were hair.

\section{Results and Discussion}

The mean RT data of Experiment 2 are shown in Table 2 (third and sixth column). A three-way ANOVA, with stimulus condition (eye or hair) and responding hand as between-group factors and with the response type ("same" or "different") as within-group factor, was calculated, the main effect of stimulus condition $[F(1,12)$ $=10.07, \mathrm{p}<.01]$ indicated shorter RT for hair than for eyes. The other significant effect was the interaction between the stimulus condition and response type $[\mathrm{F}(1,12)$ $=6.81, \mathrm{p}<.05]$. According to this interaction, in the hair-stimulus condition, the "same" RT was shorter than the "different" RT, whereas, in the eye stimulus condition, a shorter "different" RT was obtained.

The mean error data are shown in Table 3. A two-way ANOVA was calculated from the main error numbers, with stimulus condition (between group) and response type (within subject) as factors. Both main effects were significant for the stimulus condition $[F(1,14)=36.1, p<$ $.01]$. It is evident that there were fewer errors in the hair stimulus condition and in the "different" response condition.

In order to assess the effect of the irrelevant features and of the face as a whole, these comparisons were made between the results of Experiments 1 and 2. Data relevant to this comparison are given in Table 2.

The average "different" RT of Experiment 1 was compared with the "different"' RT of Experiment 2. According to a two-way ANOVA (stimulus condition $\times$ experiment), the "different"' $R T$ is shorter for hair $[F(1,32)=$ $4.78, p<.05]$. There were no other significant effects.

Table 2

Same and Different RT (in Milliseconds) in Experiments 1 and 2

\begin{tabular}{|c|c|c|c|c|c|c|}
\hline \multirow[b]{3}{*}{ Stimulus Condition } & \multicolumn{3}{|c|}{ Same } & \multicolumn{3}{|c|}{ Different } \\
\hline & \multicolumn{2}{|c|}{ Experiment 1} & \multirow[t]{2}{*}{ Experiment 2} & \multicolumn{2}{|c|}{ Experiment 1} & \multirow[t]{2}{*}{ Experiment 2} \\
\hline & Absolutely Same & Mean & & Absolutely Different & Mean & \\
\hline $\begin{array}{l}\text { Eye } \\
\text { Hair }\end{array}$ & $\begin{array}{l}633 \\
603\end{array}$ & $\begin{array}{l}794 \\
664\end{array}$ & $\begin{array}{l}742 \\
525\end{array}$ & $\begin{array}{l}662 \\
621\end{array}$ & $\begin{array}{l}670 \\
630\end{array}$ & $\begin{array}{l}699 \\
588\end{array}$ \\
\hline
\end{tabular}


Table 3

Percentage Error in Experiment 2

\begin{tabular}{ccc}
\hline Stimulus Condition & Same & Different \\
\hline Eye & 13.7 & 10.1 \\
Hair & 9.8 & 3.9 \\
\hline
\end{tabular}

In the next comparison, the "absolutely different" (i.e., four different features) condition of Experiment 1 was compared with the "different" RT of Experiment 2. Again, the only significant effect was the shorter "different" $R T$ for hair $[F(1,32)=5.19, p<.05]$. Comparing the "same" RT data of Experiment 1 and Experiment 2, certain differences are evident. According to a two-way ANOVA, the average "same" RT of Experiment 1 is longer than that of Experiment $2[\mathrm{~F}(1,32)=5.40, \mathrm{p}<$ .05]. The main effect of stimulus condition is significant as well $[\mathrm{F}(1,32)=17.87, \mathrm{p}<.01]$, indicating a shorter RT for the hair-stimulus condition. A different picture emerges when the "same" RT of Experiment 2 is compared with the "absolutely same" (i.e., zero different feature) condition of Experiment 1. In this case, the main effect of stimulus condition is significant $[F(1,32)=$ $12.80, p<.01]$. The other significant effect is the stimulus condition $\times$ experiment interaction $[F(1,32)=7.36$, $\mathrm{p}<.05]$. According to this interaction, the presence of other features of the face increased the "same" RT when the hair was the critical feature, but decreased it when the critical feature was the eye.

Given the shorter RT and the smaller number of errors, discriminability of the hair stimuli in the present experiments seems to be greater than that of the eyes. However, in the case of the two facial features investigated, the relationship between "same" and "different" RT is just the opposite. In the hair stimulus condition, a shorter "same" RT was obtained, whereas, for the eye, the "different" RT was shorter. In experiments employing line drawings of faces, Sergent (1982) did not report "same"'-"different" RT divergences. The photographic stimuli of the present study were richer in detail than the line drawings. This difference was particularly true for the eyes. This facial feature can be considered as more complex than hair. The complexity and difficulty of discrimination are factors that may favor the analytic type of processing (Kroll \& Hershenson, 1980). On the other hand, hair has definite and close contour, thus resembling the stimuli more frequently employed in matching studies, in which a "fast-same" effect was reported (Cooper, 1976; Hawkins, 1969; Miller \& Bauer, 1981; Nickerson, 1967).

At the same time, it is conceivable that the eyes, being less well-defined units, are more prone to the disturbing effects of the other facial features.

\section{EXPERIMENT 3}

The aim of this experiment was to assess the generality of the findings of Experiment 1 . In the present experiment, the standard stimulus was continuously presented during an experimental run. This way, the subjects had the opportunity to select a proper encoding strategy. In addition, they were able to simultaneously observe the stimuli to be matched.

\section{Method}

Subjects. The subjects were 16 paid students of Bárczi Gusztáv College for Teachers of Special Education, Budapest.

Stimuli and Procedure. The stimuli were the photographs used in Experiment 1. From the eight sets (four for the eye-stimulus condition and four for the hair-stimulus condition), eight stimulus runs were constructed. In each run, 24 members of the set were arranged randomly for each subject. The presentation of the stimuli of a given set was preceded by two randomly selected practice trials. The order of presentation of the sets was counterbalanced among the subjects.

By using a two-channel mirror system and a Kodak Carousel projector, the standard stimulus was projected to either the left or the right half of a screen subtending $6^{\circ}$ horizontally and $4^{\circ}$ vertically from the $120-\mathrm{cm}$ viewing distance. This standard stimulus was visible throughout the whole experimental run. The comparative stimuli were projected to the other half of the field. Stimulus duration was controlled by an electronic shutter (Getra). The comparative stimuli were presented for $1,000 \mathrm{msec}$. A warning click preceded the presentation of the comparative stimulus by $1,000 \mathrm{msec}$. The measurement of the RT was identical with that of the previous experiments.

For half of the subjects, the standard stimuli appeared on the right side; for the other half, the standard stimuli were presented on the left side. Half of the subjects responded with the right hand when they decided that the target features were "same," whereas the other half of the subjects used the right hand for "different" responses.

\section{Results and Discussion}

The main RT data are shown in Figure 3. Separate three-way ANOVAs were calculated for the "same" and "different" responses, with stimulus location (right or left side of the standard stimulus) as a between-group factor and stimulus condition (hair or eye) and the number of different features (four levels) as within-subjects factors. Concerning the responding hands, the data were pooled. In the case of the "same" stimuli, the main effect of stimulus type and the number of different features appeared to be significant $[\mathrm{F}(1,14)=17.31, \mathrm{p}<.01$, and $F(3,42)=28.33, p<.01$, respectively]. A significant response type $\times$ number of different feature interaction appeared as well $[F(3,42)=11.66, p<.01]$. A similar analysis on the "different" RT indicated a significant main effect of stimulus type only $[\mathrm{F}(1,14)=12.11, \mathrm{p}<.01]$.

In order to analyze the effect of the number of different features, separate one-way ANOVAs were calculated for the eye- and hair-stimulus conditions. For the eyes, this yielded $F(3,45)=26.23, p<.01$, and for the hair $F(3,45)=11.01, p<.01$. In both cases, only the linear trend was significant [for eyes, $F_{\text {lin }}(1,45)=77.99, p<$ .01 ; for hair, $\left.F_{\text {lin }}(1,45)=28.65, p<.01\right]$.

In comparing the "same" and "different" RTs, responses were collapsed for the number of different irrelevant features, and a three-way ANOVA was calculated, with stimulus location as a between-group factor and response type and stimulus condition as withinsubjects factors. The main effects of response type and 


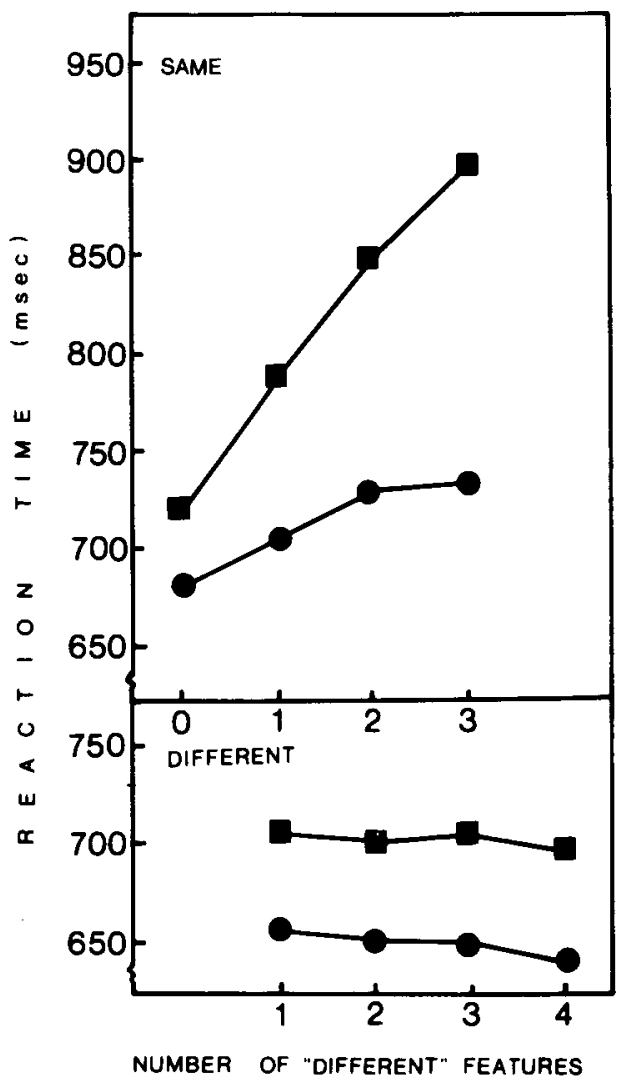

Figure 3. Mean "same" and "different" RT as function of the number of different features in Experiment 3. $\square-0$, eye target; $\bullet-\bullet$, hair target.

stimulus condition appeared to be significant $[F(1,14)=$ $18.00, \mathrm{p}<.01$, and $\mathrm{F}(1,14)=17.58, \mathrm{p}<.01$, respectively]. There was a significant response type $\times$ stimulus condition interaction as well $[F(1,14)=9.27, p<.01]$.

The main percentages of errors are shown on Table 4 . A three-way ANOVA was calculated for the main error numbers. Stimulus location was a between-group factor and stimulus condition and response type were withinsubject factors. The main effect of the latter two factors appeared to be significant [for stimulus condition, $F(1,14)$ $=14.08, \mathrm{p}<.01$; for response type, $\mathrm{F}(1,14)=6.20$, $\mathrm{p}<.05]$.

The present data are consistent with the main findings of Experiment 1. As the number of the irrelevant different features increased, the "same" RT also increased. The rate of the increase was larger in the eye stimulus condition. As in Experiment 1, the number of irrelevant

Table 4

Percentage Error in Experiment 3

\begin{tabular}{|c|c|c|c|c|c|c|c|c|}
\hline \multirow[b]{3}{*}{ Stimulus Condition } & \multicolumn{4}{|c|}{ Same } & \multicolumn{4}{|c|}{ Different } \\
\hline & \multicolumn{4}{|c|}{ Different Features } & \multicolumn{4}{|c|}{ Different Features } \\
\hline & 0 & 1 & 2 & 3 & 1 & 2 & 3 & 4 \\
\hline Eye & 7.8 & 8.7 & 6.4 & 6.8 & 7.3 & 7.4 & 7.8 & 3.1 \\
\hline Hair & 4.7 & 3.2 & 3.2 & 1.6 & 0.0 & 2.1 & 3.6 & 1.1 \\
\hline
\end{tabular}

different features has no effect on the "different" RT. Although the stimulus condition has no effect on the "different" RT in Experiment 1, both the "same" and the "different" RT appeared to be shorter for the hair stimulus condition than for the eye stimulus condition in the present experiment. As in Experiment 1, "different" RT was shorter than "same" RT and the "same"-" different" divergence was larger for the eye than for the hair stimuli. The distribution of errors was similar in the two experiments. According to the results, under the more relaxed conditions of Experiment 3, that is, with the continuous presentation of the standard stimuli throughout an experimental run, and with the possibility of simultaneous consideration of the to-be-matched stimulus pairs, the results of Experiment 1 were either replicated or amplified.

The "fast-same" effect is usually larger with successive presentation (Krueger, 1983; Proctor, 1981), or successive presentation is even a necessary condition for the emergence of the effect (Moscovitch et al., 1976). The effect of different irrelevant features on the "same" RT, however, is not confined to memory-matching conditions.

\section{GENERAL DISCUSSION}

Recent analyses of matching tasks emphasize the importance of multiple considerations of the stimulus representations (Kroll \& Hershenson, 1980; Krueger, 1979), mutual inhibitory relationships between potential responses (Eriksen et al., 1982; Proctor, 1981), the effects of information load on decision mechanisms (Miller \& Bauer, 1981), or the importance of holistic and analytic processes (Sergent \& Bindra, 1981). Although these approaches are frequently regarded as competing theories (e.g., Bagnara et al., 1983), closer investigation suggests that basic principles underlying any single approach are not really incompatible with the assumptions underlying other approaches.

One of the main findings of the present study, that is, the linear increase of the "same" RT as a function of the number of irrelevant different features, is in agreement with the response competition approaches (Eriksen et al., 1982; Proctor, 1981). However, in order to account for some details of the present results, one must consider the effects of the formation of holistic facial representation and the necessity of spatial selection as well.

In the task of Experiments 1 and 3, the subjects had to attend selectively to the task-relevant feature. As indicated by the low error rate, the subjects were quite successful. The mechanism of selection was, however, neither perfect nor homogeneous. The presumed reason for this is the joint effect of irrelevant features on the "same" RT, together with the different magnitude of this effect on the eye and hair stimulus conditions. The overall advantage of the hair stimulus condition may be attributable to the better discriminability of this feature. However, it should be mentioned that a reasonable explanation for the increased effect of the irrelevant features 
on eyes might be sought in the fact that the stimulus set kind of selection (Broadbent, 1970) is less effective in the eye stimulus condition than in the hair stimulus condition. If the filter mechanism is less effective for the eyes, the effect of disturbing features increases the load on the decision-making mechanism. In order to overcome the effect of the disturbing features, a selection process more elaborate than stimulus set is needed.

As for the assumption of more effective filtering in the hair stimulus condition, one might argue that, in the case of this more integrated, more independent feature, the cost of filtering is higher (Treisman, Kahneman, \& Burkell, 1983). Thus, the filtering should increase the RT to a larger extent in the hair stimulus condition than in the eye stimulus condition. The filtering cost effect has been demonstrated, however, in connection with perceptual organization (Treisman et al., 1983). In the case of the present stimuli, the possibility of the action of peripheral mechanisms (see Discussion of Experiment 1) may counteract the effect of the cost of filtering.

The unitary character of the face is a peculiar one. It is the physiognomy, the identity, of a person. The contribution of the eyes is essential for this kind of identity (Davies et al., 1977; Laughery et al., 1971). In addition to the contribution made by perceptual configuration phenomena, this functional categorization (Sergent \& Bindra, 1981) may contribute to the unitary representation of faces. In fact, the face as a whole exerted a complex effect on RT in the present study. In the presence of other parts of the face, the "different" RT was shorter than the "same" for both relevant features. When the features were presented in isolation, however, the "different" RT remained shorter only in the eye stimulus condition. Furthermore, in the presence of the face as a whole, the shortest "same" RT (i.e., when identical faces were presented) was shorter than the "same"' RT for isolated eyes. In the hair stimulus condition, the opposite result was obtained.

In order to interpret this difference, it is necessary to reconsider the effect of the unitary character of facial representation. The subjects had to concentrate on a given facial feature. Due to imperfect filtering, the decision mechanism in the hair stimulus condition dealt with some irrelevant features as well. As a function of the number of the obtained differences, the priming of the "different" response may increase; and, as suggested by response competition models (Eriksen et al., 1982; Proctor, 1981), the "same" RT increases due to the emergence of inhibitory processes. Concentrating on the eyes, however, the formation of a holistic representation might be more compelling. In order to maintain a low error rate, in the vast majority of experimental trials the subjects had to avoid reacting on the basis of the holistic representation. Maintaining the principles of the continuous flow models (Eriksen et al., 1982; Eriksen \& Schultz, 1979; McClelland, 1979), the recognition response inhibition model (Proctor, 1981), or even the "asynchronous dis- crete coding" approach (Miller, 1982), the results of the holistic processes might exert a priming effect on the reaction organization. The priming of the "same" responses would be largest in the case of identical pairs of faces. Accordingly, the "same"' RT in this case might be faster than the "same" RT to isolated eyes (Experiment 2).

A somewhat puzzling result of the present study is the insensibility of "different" RT to the number of same/different irrelevant features. As was suggested, the present RT and error data did not rule out the possibility of a "floor effect." In fact, in more than $85 \%$ of the trials, the stimuli were actually different. The almost permanent priming of the "different" response may explain the advantage of the "different" response. This priming may contribute to a response bias towards the "different" response. The direction of this supposed bias, however, is just the opposite of the response bias reported in some matching experiments (e.g., Ratcliff \& Hacker, 1981).

In conclusion, it is important to emphasize that the eclectic flavor evident in the discussion of the present results is a consequence of the complexity of the stimulus material utilized in these experiments. The potential for explanations of this kind, however, is dependent on the elaboration of models developed to explain the phenomena of matching tasks.

\section{REFERENCES}

Bagnara, S., Boles, D. B., Simion, F., \& Umiltà, C. (1983). Symmetry and similarity effects in the comparison of visual patterns. Perception \& Psychophysics, 34, 578-584.

BAmber, D. (1969). Reaction time and error rates for "same" "- different" judgments of multidimensional stimuli. Perception \& Psychophysics, 6, 169-174.

Broadbent, D. E. (1970). Stimulus set and response set: Two kinds of selective attention. In D. I. Mostofsky (Ed.), Attention: Contemporary theory and analysis. New York: Appleton.

Christie, D. F. M., \& Ellis, H. D. (1981). Photofit constructions versus verbal description of faces. Journal of Applied Psychology, 66, 358-363.

CoHEN, G. (1972). Hemispheric differences in letter classification task. Perception \& Psychophysics, 11, 139-142.

COOPER, L. A. (1976). Individual differences in visual comparison processes. Perception \& Psychophysics, 19, 433-444.

Davies, G., Ellis, H. D., \& ShePherd, J. W. (1977). Cue saliency in faces as assessed by the "Photofit" technique. Perception, 6, 263-269.

Dixon, P., JusT, M. (1978). Normalization of irrelevant dimensions in stimulus comparisons. Journal of Experimental Psychology: Human Perception and Performance, 4, 36-46.

Ellis, H. D., Shepherd, J. W., Davies, G. M. (1975). An investigation of the use of the Photofit technique for recalling faces. British Journal of Psychology, 66, 29-37.

Eriksen, C. W., OHARA, W. P., ERIKSEN, B. (1982). Response competition effects in same-different judgments. Perception \& Psychophysics, 32, 261-270.

ERIKSEN, C. W., \& SChULTZ, D. W. (1979). Information processing in visual search: A continuous flow conception and experimental result. Perception \& Psychophysics, 25, 249-263.

Hawkins, H. (1969). Parallel processing in complex visual discrimi nation. Perception \& Psychophysics, 5, 56-64.

Kroll, J. F., \& Hershenson, M. (1980). Two stages of visual matching. Canadian Joumal of Psychology, 34, 49-61. 
KrUeger, L. (1978). A theory of perceptual matching. Psychological Review, 85, 278-304.

KRUEGER, L. E. (1979). A model of unidimensional perceptual matching. Journal of Experimental Psychology: Human Perception and Performance, 5, 277-288.

Krueger, L. E. (1983). Probing Proctor's priming principle: The effect of simultaneous and sequential presentation on same and differential judgments. Joumal of Experimental Psychology: Learning, Memory, and Cognition, 9, 511-523.

Laughery, K. R., Alexander, J. F., LANe, A.B. (1971). Recognition of human face: Effect of target exposure time, target position, and type of photograph. Journal of Applied Psychology, 55, 479-483.

MCClelland, J. (1979). On the time relations of mental processes. Psychological Review, 86, 287-330.

Miller, J. (1982). Discrete versus continuous stage models of human information processing: In search of partial output. Joumal of Experimental Psychology: Human Perception and Performance, 8, 273-296.

Miller, J., \& BAUER, P. W. (1981). Irrelevant differences in the "same"'-"different"' task. Journal of Experimental Psychology: Human Perception and Performance, 7, 196-207.

Moscovitch, M., Scullion, D., \& ChRISTIE, D. (1976). Early versus late stages of processing in their relation to functional asymmetries in face recognition. Joumal of Experimental Psychology: Human Perception and Performance, 2, 401-416.

NiCKE RSON, R. (1967). "Same"-"different" response times with multiattribute stimulus differences. Perceptual and Motor Skills, 24, 543-554.

Patterson, K., \& Bradshaw, J. C. (1975). Differential hemispheric mediation of nonverbal visual stimuli. Journal of Experimental Psychology: Human Perception and Performance, 1, 246-253.

PROctoR, R. W. (1981). A unified theory for matching-task phenomena. Psychological Review, 88, 291-326.

RATCLIFF, R., \&ACKER, M. J. (1981). Speed and accuracy of same and different responses in perceptual matching. Perception \& Psychophysics, 30, 303-307.

SERGENT, J. (1982). About face: Left-hemisphere involvement in processing physiognomies. Journal of Experimental Psychology: Human Perception and Performance, 8, 1-14.

SERGENT, J., \& BindRA, D. (1981). Differential hemispheric processing of faces: Methodological considerations and reinterpretation. Psychological Bulletin, 89, 541-554.

SUbERI, M., \& MCKeEver, W. F. (1977). Differential hemispheric memory storage of emotional and non-emotional faces. Neuropsychologia, 15, 757-768.
Treisman, A., Kahneman, D., \& Burkell, J. (1983). Perceptual objects and the cost of filtering. Perception \& Psychophysics, 33, 527-532.

YARBUS, A. L. (1967). Eye movement and vision. New York: Plenum.

\section{NOTES}

1. In the eye stimulus condition $t(9)=1.50$, and in the hair stimulus condition $t(9)=1.31$. In both cases $p>.1$.

2 . In the scaling experiment, 16 subjects rated the similarity of each stimulus to the standard of the given set. Half of the subjects rated the stimuli of the eye stimulus condition, and the other half rated the stimuli of the hair stimulus condition. There were 12 "same" and 12 "different" pictures in each set. Thus, the subjects rated 48 pictures. The number of different features were $0,1,2$, or 3 for the "same" pictures and $1,2,3$, or 4 for the "different" pictures. On a 7-point scale, the lower values indicated higher similarity. The average scale values of the "same" stimuli were $1.4,3.0,3.9$, and 4.2 in the eye stimulus condition and $1.3,3.0,4.2$, and 5.1 in the hair stimulus condition. The scale values of the "different" stimuli were 4.0,4.8, 5.5, and 6.0 in the eye stimulus condition and $3.6,4.7,5.4$, and 6.0 in the hair stimulus condition. In a three-way ANOVA, the scale values of the common levels $(1,2$, or 3$)$ of the "same" and "different" stimuli (within-subject factors) and the two stimulus conditions (between-subject factor) were compared. The main effect of response type $[F(1,14)=15.75]$ and number of different features $[F(2,28)=51.24]$ appeared to be significant. In both cases, $\mathrm{p}<.01$. A significant three-way interaction $[\mathrm{F}(2,28)=$ $6.82, \mathrm{p}<.01]$ was found as well. According to separate one-way ANOVAs, the effect of the number of different features was significant in both stimulus conditions and for both response types $[\mathrm{Fs}(3,21)=$ 103.73 and 28.40 in the eye stimulus condition for the "same" and "different" RT, respectively, and $F s(3,21)=35.95$ and 39.60 in the hair stimulus condition for the "same"" and "different" RT, respectively]. In all cases $p<.01$. Significant linear trends $(p<.01)$ were found in all cases. Thus, the "same" stimuli were more similar to the standard than the "different" ones, but the subjects were able to discriminate between the similarity levels in the cases of both types of stimuli. Concerning the results of Experiment 1 and the scaling experiment, in the hair stimulus condition the decrease of similarity from 4.2 to 5.1 corresponded to more than a $60-\mathrm{msec}$ change in the "same" RT. On the other hand, the decrease of similarity from 3.6 to 5.4 corresponded to no change for the "different" RT.

(Manuscript received August 21, 1984; revision accepted for publication January 30,1985 .) 